\title{
COMUNIDAD ESTACIONAL DE MICROHONGOS EN LA LITERA DE CONIFERAS DEL N.E. ARGENTINO: ENFASIS EN TAXA DE POTENCIAL INTERES MEDICO
}

(Seasonal microfungal community of coniferous litter of Argentine N.E: emphasis on potential medical interest taxa)

\author{
Eduardo Piontelli*, Gustavo Giusiano** \& Dunny Casanova *** \\ *Univ. deValparaíso, Escuela de Medicina, Cátedra de Micología, Casilla 92 V Valparaíso, Chile \\ **Universidad del Nordeste, Inst. Med. Reg. Dep. Micología \\ Av. Las Heras 727, 3500 Resistencia (Chaco), Argentina \\ *** Universidad de Valparaíso Esc. de Medicina, Departamento de Salud Pública
}

Palabras clave:Microhongos oportunistas, litera de coníferas, salud pública.

Key words:Opportunist microfungi, coniferous litter, public health.

\section{RESUMEN}

Se analizó la colonización in vitro de microhongos filamentosos de rápido crecimiento en la litera de coníferas (Pinus elliottii) en el N.E argentino, mediante muestras estacionales de acículas senescentes de 4 localidades con semejanzas climáticas, edáficas y vegetacionales. Veinte muestras (5 de cada lugar) se sembraron en agar papa dextrosa y agar agua (ambos adicionados con dichlorán) durante 21 días a $t^{\circ}$ ambiente, para enumerar la presencia relativa de su micota. Se contabilizó un total de 63 géneros fúngicos y 117 especies, representadas mayoritariamente por anamorfos de Ascomycota (85\%), Ascomycota (9,9\%) y Zygomycota (4,1\%). Los hongos dematiáceos fueron dominantes $(81,9 \%)$ y los 5 géneros mas frecuentes y constantes en las 4 estaciones y localidades, presentaron un variado comportamiento estacional, tales como: Cladosporium (31,8\%), Alternaria (13,5\%), Epicoccum (8,8\%), Trichoderma (5,7\%) y Fusarium (4,9\%), con el $65 \%$ de la presencia total. Los géneros con mayor variedad de especies fueron: Curvularia (7), Fusarium (6), Phoma (6), Trichoderma (5) y Cladosporium (4). Las especies de interés médico fueron 52, con una presencia del 73,3\% de la micota total. Las frecuencias más altas fueron: A.alternata, A.tenuisssima, Bipolaris cynodontis, C.cladosporiodes, C.oxysporum, Curvularia lunata, E. nigrum, F. semitectum, Nigrospora sphaerica, T. harzianum y T.viride.

\section{INTRODUCCION}

A partir de uno de los pioneros trabajos de Kendrick \& Burges (1962), las comunidades de microhongos en
ABSTRACT

The in vitro colonization of fast growth filamentous microfungi in the coniferous litter (Pinus elliottii) in northern-eastern Argentina was analysed by using seasonal samples of senescent acicules from four localities showing similar climatic, edaphic and vegetational characteristics. Twenty samples (five from each site) were cultured in dextrose potato agar and water agar (both dichloran added) for 21 days at room temperature in order to assess the relative presence of their mycota. A total sixty three fungal genera and 117 strains were detected, most of them being anamorphs of Ascomycota (85\%), Ascomycota (9.9\%) and Zygomycota (4.1\%). Dematiaceous fungi were dominant (81.9\%) whereas the five genera most frequent and constant in the four stations and localities showed a varied seasonal behaviour such as:Cladosporium (31.8), Alternaria (13.5\%), Epicoccum (8.8\%), Trichoderma (5.7\%) and Fusarium (4.9\%) with $65 \%$ of the entire presence. Genera bearing a higher variety of species were: Curvularia(7),Fusarium (6), Phoma (6), Trichoderma (5) and Cladosporium (4). Species showing medical interest were 52, with a $73.3 \%$ presence of the total mycota. The highest frequencies were:A:alternata A.tenuisssima, Bipolaris cynodontis, C. cladosporiodes, C.oxysporum, Curvularia lunata, E. nigrum, F.semitecnum, Nigrospora sphaerica, T.harzianum and T.viride.

acículas de coníferas senescentes se han estudiado desde varios puntos de vista en muchos continentes, principalmente analizando sus roles ecológicos, su sucesión, la variación de su distribución geográfica y la presencia de fitopatógenos (Groubière, 1974; Miller, 1974; Black \& Dix, 
1977; Minter, 1981; Takayuki \& Tokumasu, 1995; Tokumasu, 1998; Groubier \& Debouzie, 2003).

Se han observado grandes diferencias en la constancia y abundancia de los hongos dominantes en acículas senescentes al caer estas en la litera, principalmente por la competencia de muchos saprófitos del suelo de rápido o lento crecimiento (Horizonte $\mathrm{O}$ ), que imponen a la comunidad varios estados en la sucesión de especies (Tokumasu \& Aoiki, 2002; Jones \& Hyde, 2002).

Las acículas de coníferas constituyen una pequeña unidad de recursos y sus poblaciones fúngicas solo pueden cuantificarse acorde a la proporción de unidades que originan cuerpos fructíferos (Rayner, 1994; Zak \& Rabatin, 1997). Los problemas en la caracterización de especies en los ecosistemas terrestres radican principalmente en determinar su número sobre un determinado substrato, debido a que mientras algunas se presentan como un único cuerpo fructífero, otras lo hacen sobre gran parte del substrato creando problemas en la enumeración y cuantificación en los modelos estadísticos (Jones \& Hyde, 2002).

La colonización de la litera de coníferas soporta una micota saprofítica característica que muestra ciertas diferencias poblacionales si se compara con la presente en las angiospermas (Hudson 1968; Black \& Dix, 1976). La presencia de fenoles y ácidos ferúlicos en el substrato, ejercen indudablemente un efecto estimulante o inhibidor para el crecimiento de algunos hongos del suelo o epifitos de la filósfera, especialmente en la colonización de los tejidos internos (Black \& Dix, 1976).

Para su análisis se han usado varias metodologías que van desde la obsevación directa bajo el microscopio, incubación en cámara humeda, lavado, siembra en medios de cultivos diversos, la impresión directa de las hojas en el medio de cultivo, la microscopía electrónica (Scanner) y la esterilización superficial (endófito), entre otras ( Parkinson, 1982; Andrews \& Hirano, 1991; Bills \& Polishook. 1994).

No hemos encontrado aparentemente en la literatura trabajos relacionados con la micota de la litera de coníferas (Pinus elliottii) en el nordeste Argentino y nuestro interés se centró básicamente en un análisis estacional de la diversidad de especies de rápido crecimiento y dispersión aérea desde este substrato, con la finalidad de enumerar la presencia relativa de aquellos taxa de interés clínico (oportunistas), capaces de causar morbilidad y hasta mortalidad en pacientes con previas enfermedades graves o con compromiso de sus defensas. El espectro de las capacidads patogénicas fúngicas continúa evolucionando en el tiempo (hongos emergentes), en especial en el aumento de las poblaciones de hospedadores con compromiso inmune, enfermedades crónicas o expuestos a ciertas condiciones ambientales (espacios internos y externos), donde la inhalación, el contacto con tejidos dañados o el trauma directo, son la puerta de entrada de estos microorganismos con gran capacidad de dispersión aérea. Nuestros objetivos fueron:

1) Establecer la dominancia y constancia de especies fúngicas capaces de esporular en acículas senescentes de la litera mediante el empleo de 2 técnicas de cultivo en un muestreo estacional en bosques de coníferas en el nordeste argentino.

2) Determinar en la estructura de la comunidad la frecuencia de presencia de especies consideradas potencialmente patógenas o alergénicas para el hombre.

\section{MATERIALES Y METODOS}

1.- Datos geográficos. El área estudiada se encuentra ubicada en la zona de Misiones, entre los paralelos 25 $28^{\prime}$ y $28^{\circ} 10^{\prime}$ Latitud Sur y los meridianos $53^{\circ} 38^{\prime}$ y $56^{\circ} 03^{\prime}$ Longitud Oeste de la región nordeste de la República Argentina, atravesada por varios ríos y constituyéndose en una de las provincias más humedas del país. Tiene un clima subtropical húmedo, sin períodos secos con lluvias constantes y regulares durante todo el año (1800 mm), pero principalmente en primavera y verano. Por su cercanía al trópico de Capricornio, presenta veranos muy húmedos y calurosos e inviernos templados con temperaturas medias anuales de $20^{\circ} \mathrm{C}$ y mayores de $30^{\circ} \mathrm{C}$ en la época estival. (Area de zona de muestreo en Fig. 1).

2.-Recolección de las muestras. La recolección del material se efectuó 4 veces en el año, uno en la mitad de cada estación iniciándose en el otoño del año 2003 hasta la primavera del 2004.

Se recolectaron en cada estación sólo acículas enteras de tonalidades de color café, ubicadas principalmente en la superficie de la litera y desde 4 puntos de recolección diferentes, distanciados unos $30 \mathrm{~km}$ uno del otro. En cada una de la 4 áreas se calculó una extensión aproximada de $1000 \mathrm{~m}^{2}$ donde se marcaron al azar entre los pinos (en los limites de estas y en el centro) 5 diferentes sub áreas delimitadas con estacas en una superficie de aproximadamente $30 \mathrm{~m}^{2}$, para que todos los muestreos fueran en el tiempo, siempre en los mismos lugares. De esta manera, las 5 muestras de las 4 áreas geográficas analizadas en cada estación incluyeron 20 muestras que constituyeron el total de cada período de recolección. Debe especificarse que no se compararon entre si las 4 áreas de muestreo debido a la uniformidad climática, edáfica y de su flora en los puntos muestreados y por ser un solo muestreo por estación. En bolsas de polietileno estériles se transportó el substrato a cultivar (aproximadamente unas 60-90 acículas por muestra). Después de la colecta, éstas se mantuvieron refrigeradas en el laboratorio $\left(4-6^{\circ} \mathrm{C}\right)$ hasta su procesamiento.

3.-Medios de cultivos usados y técnica de siembra. Se em- 


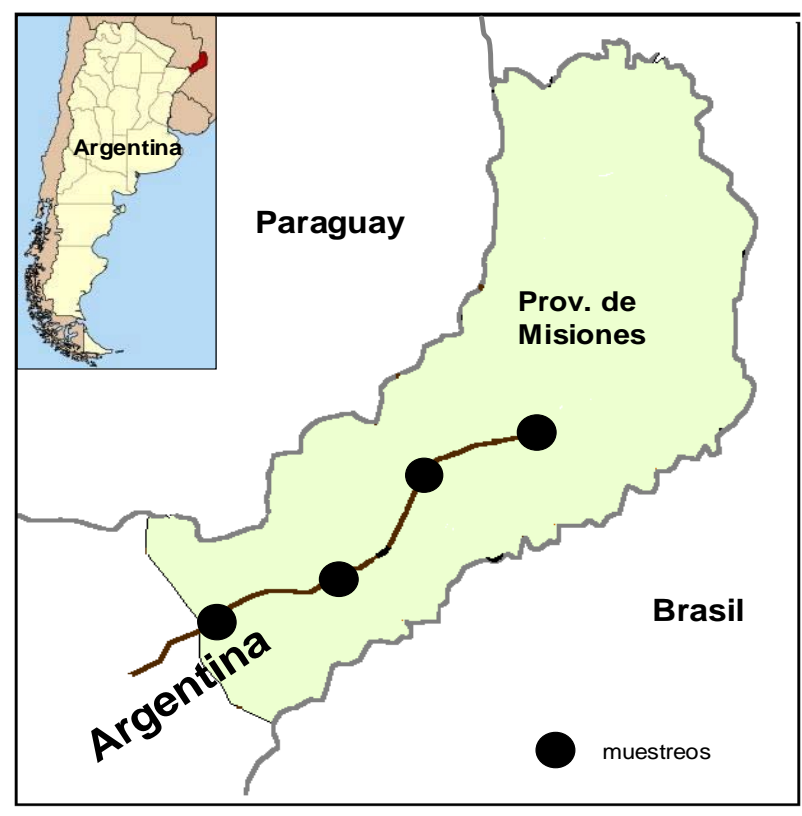

Figura 1. Area de muestreo y localización geográfica

plearon dos técnicas de cultivo: a) cultivo directo en placa de las acículas en agar papa-zanahoria (PZ) + 0,0025g/L de Dichloran, para restringir el crecimiento de las colonias fúngicas y 250mg Cloranfenicol /L ; b) cultivo directo en placas de las acículas en agar agua con Dichloran (como cámara húmeda). Cada siembra se preparó de la siguiente manera: considerando el largo de las acículas, éstas se cortaron a un tamaño de unos $3-5 \mathrm{~cm}$ y luego se lavaron 3 veces en matraces de vidrio vertiendo sobre estas agua estéril. Los matraces se ocluyeron en su parte superior con una malla metálica para dejar fluir el exceso de agua sin que escurrieran las acículas. Luego se secaron a $35^{\circ} \mathrm{C}$ durante 3-4 horas colocándolas lo más separado posible en placas de vidrio estériles. Con estos procedimientos se eliminó en parte, las impurezas superficiales como tierra y propágulos libres para dejar principalmente las estructuras fúngicas adheridas o que colonizaban el substrato.

En cada medio de cultivo se colocaron 8 acículas tomadas al azar disponiendose en forma más o menos paralela y equidistantes en la superficie de ambos medios para facilitar la detección y enumeración de los taxa.

La frecuencia de presencia de las fructificaciones de cada taxa se contabilizó en ambos medios de cultivo sólo una vez por acícula no importando que éste se repitiera más veces en la misma. De esta manera cada taxa en una placa de cultivo se enumeró del 0 al 8, patrón que se empleó en la suma en las 20 muestras por estación.

Todos los cultivos (en todas las estaciones) se incubaron a temperatura ambiente entre $18-25^{\circ} \mathrm{C}$ durante 21 días y la presencia y determinación de los taxa en las acículas fue mediante la lupa estereoscópica (20-40x) y preparaciones teñidas con lactofenol y azul de algodón. La literatura de apoyo se basó principalmente en los textos de Ellis 1970-1976, Domsh et al,1983, Ellis \& Ellis 1985, Sutton 1980, Naj-Rag, 1993, Sivanesan, 1987. La determinación de las especies de los géneros Aspergillus, Fusarium, Penicillium y Trichoderma se efectuaron en medios de cultivos específicos acorde a sus monografías (Klich, 2002, Nelson et al, 1983; Pitt, 2000; Gams \& Bisset ,1998).

4.- Análisis estadístico. En nuestro estudio se emplearon los sigientes análisis estadísticos: Diversidad de Shannon Winner, el índice de similitud de Jaccard, el índice de asociación $\mathrm{X}^{2}$ y pruebas de significación para asociaciones entre grupos de taxa.

\section{RESULTADOS}

1.- Micota total. La composición total de la comunidad fúngica en ambos medios de cultivo y su presencia relativa en todas las estaciones, arrojó un $\mathrm{n}=4985$, con un $58,8 \%(n=2931)$ en agar PZ y un $41,2 \%(n=2054)$ en agar agua (Tabla 1). Se contabilizó un total de 63 géneros fúngicos y 117 especies, de las cuales 64 se presentaron en ambos medios, 37 crecieron sólo en agar papa zanahoria, y en agar agua sólo lo hicieron 16 (Tabla 1). La presencia fúngica en agar agua en las 4 estaciones fue menor en invierno subiendo progresivamente desde invierno a otoño, mientras en agar PZ fue menor en otoño y verano subiendo en invierno y primavera (Tabla 1, Figura 2). Los grupos taxonómicos fueron representados mayoritariamente por taxas anamorfos de Ascomycota con conidios exógenos sobre conidióforos (71\%), siguiendo en importancia los anamorfos de Ascomycota con conidióforos en cuerpos fructíferos (picnidios o acérvulos) (14\%), Ascomycota (9,9\%) y Zygomycota (4,1\%) (Tabla 1).

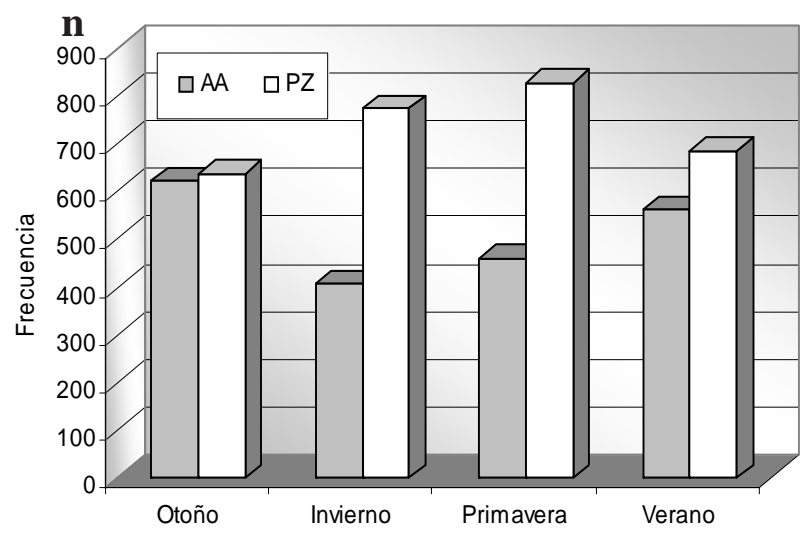

Fig. 2. Frecuencia estacional total de hongos en ambos medios de cultivo. 


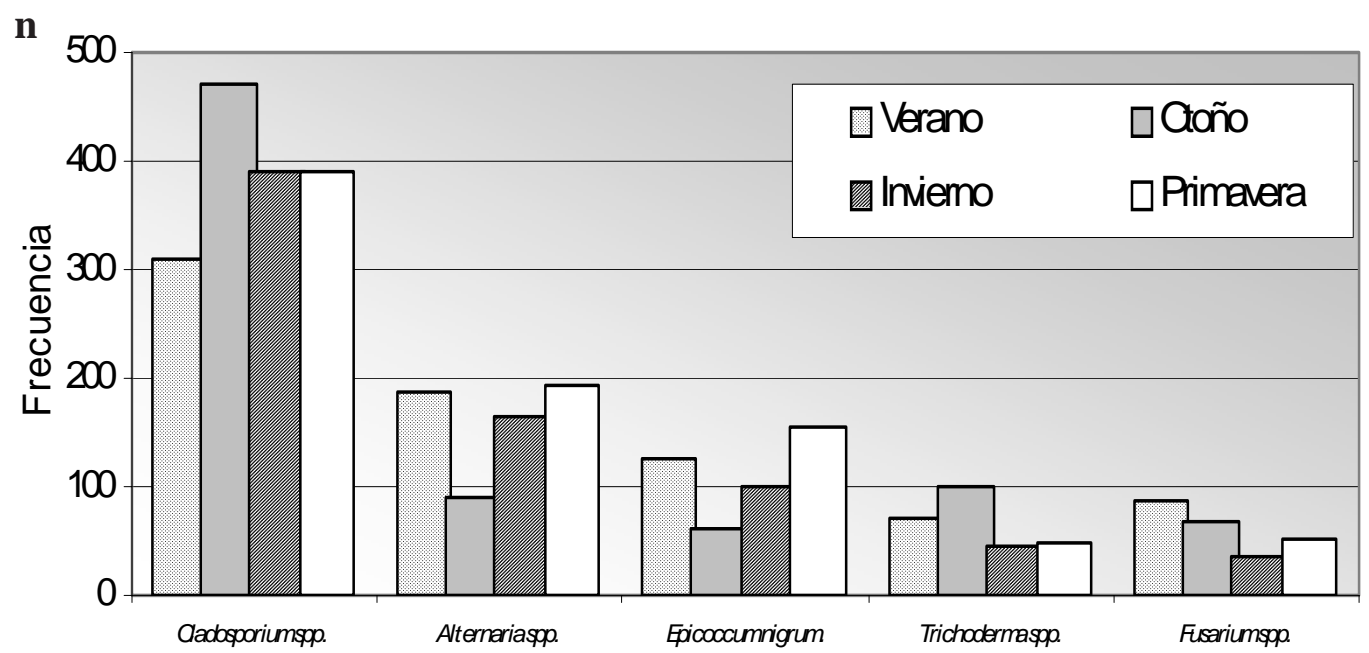

Figura.3 Taxas más frecuentes (\%) en ambos medios según estación

Los anamorfos de Ascomycota presentaron mayoritariamente 3 modalidades de ontogenia conidial; de éstos, los taxa con mayor presencia numérica (48,7\%), fueron los que presentaron una conidiogénesis blástica y conidios secos (21,5\% de los taxa), siendo los más representativos del grupo (80,8\%), destacándose las especies de Cladosporium. Los géneros con conidiogénesis fialídica con o sin conidiomas fueron mayoritarios (46,2\%), de los cuales un $21,4 \%$ presentó conidios secos ( $5,4 \%$ de presencia en el total de taxa) y un $78,6 \%$ con conidios húmedos (24,1\% del total de presencia), destacándose Trichoderma y Fusarium. Siguen en importancia los taxa con conidiogénesis trética (poroconidios) con una presencia del $17,3 \%$ en la micota, representados principalmente por Alternaria, Curvularia y Bipolaris.

Un total de 26 géneros fueron constantes en su presencia en las 4 estaciones, mientras 43 fueron esporádicos o poco comunes, presentándose una sola vez en el tiempo (Tabla 1).

Los géneros con mayor variedad de especies fueron : Curvularia (7), Fusarium (6), Phoma (6), Trichoderma (5) y Cladosporium (4). Los hongos dematiáceos dominaron ampliamente sobre los hialinos con un 81,9\% del total de presencia y los 5 géneros mas frecuentes detectados en las 4 estaciones en ambos medios fueron: Cladosporium (31,8\%), Alternaria (13,5\%), Epicoccum (8,8\%), Trichoderma (5,7\%) y Fusarium (4,9\%), los cuales representaron prácticamente el $65 \%$ de la presencia fúngica total (Tabla 1, Figura 3). Los integrantes del género Alternaria y Epicoccum nigrum tienen perfiles temporales similares (no significativamente diferentes al 1\%), presentándose ambos con un peak en primavera (Fig. 5). Por el contrario, los perfiles de las especies de Fusarium y Trichoderma, que presentan peak en otoño y verano (el primero más en otoño y el segundo más en verano). Final- mente, los integrantes de Cladosporium, se parecen más a estos dos últimos, especialmente al último, pues presenta también un peak en otoño (Fig. 5).

Los restantes taxas fueron mayoritariamente esporádicos salvo 10 de ellos que tuvieron porcentajes de frecuencias menores del $5 \%$ pero superiores al $1 \%$, tales como: Pestalotiopsis maculans, Phoma spp., Nigrospora spp., Lophodermium pinastri, Harzia spp., Aureobasidium pullulans, Pithomyces chartarum, Clonostachys rosea, Curvularia spp. y Bipolaris cynodontis (Tabla 1). Los taxa más constantes (presentes en todas las estaciones) corresponden a los mismos que obtuvieron porcentajes de presencia superiores al $1 \%$, (destacados en fondo gris en Tabla 1), los con porcentajes menores de presencia fueron más constantes sólo en agar PZ.

2.- Micota de interés clínico. La presencia total de hongos considerados de interés clínico según la literatura

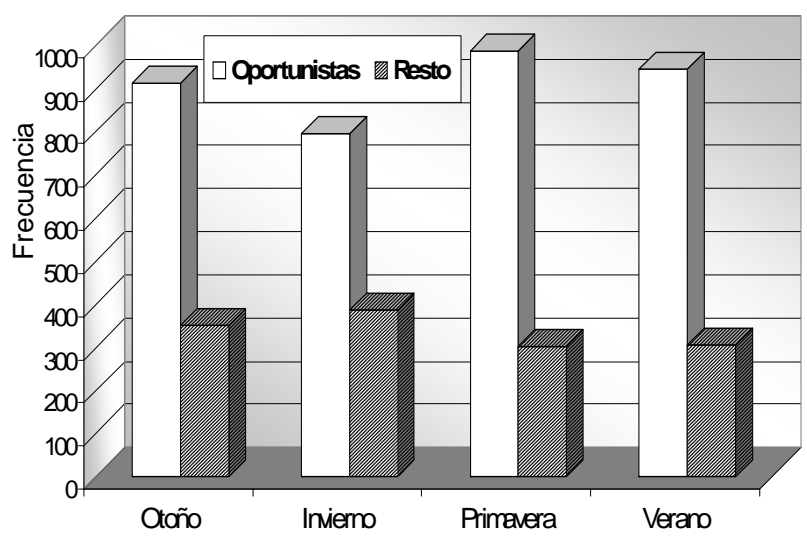

Fig. 4. Frecuencia estacional total de hongos considerados de interés clínico, en ambos medios de cultivo 


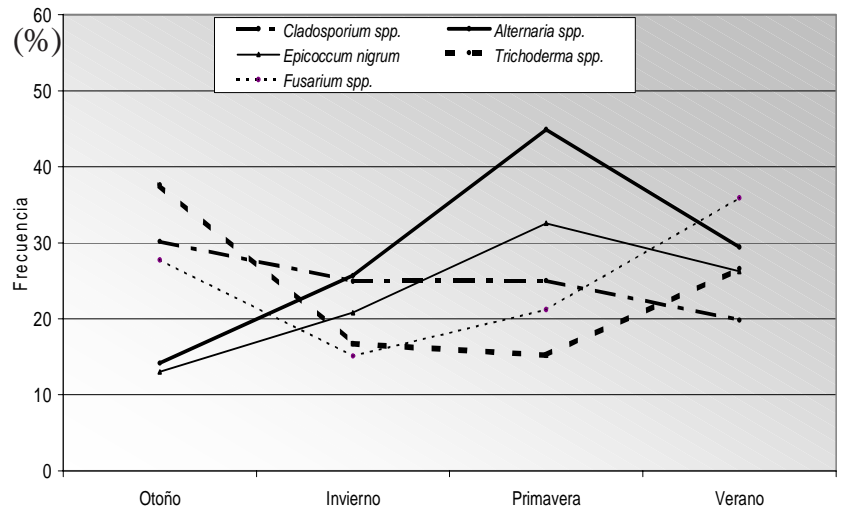

Figura 5.- Frecuencia relativa (\%) de los 5 principales taxa fúngicos según estación en ambos medios

$(n=3702$ ) fue de un $74,3 \%$ con respecto al resto de la micota presente, siendo en general los mismos con mayor constancia en Tabla 1. El 68\% de ellos fructifican mediante la producción de mitosporas secas de dispersión anemófila. La frecuencia estacional de éstos en ambos medios de cultivo fue siempre mayor en agar papa zanahoria, con leve disminución en invierno, mientras en agar agua tuvo un comportamiento muy similar en todas las estaciones, pero levemente mayor en invierno.
La mayor dominancia de taxa en ambos medios y estaciones, estuvo tambien representada por integrantes de los géneros Cladosporium, Alternaria, Epicoccum, Trichoderma y Fusarium (Tabla 1). El comportamiento estacional de los principales taxa se aprecia en Figura 4, donde Alternaria spp.y Epicoccum nigrum, presentan una misma curva de comportamiento estacional con su mayor presencia en primavera, al igual que Fusarium spp. y Trichoderma spp. en otoño y verano, disminuyendo en invierno y primavera. Debe destacarse que $\boldsymbol{F}$. semitectum fue constante todo el año y su alta presencia en otoño se ve aumentada por la presencia de otras especies. Cladosporium spp., presenta un comportamiento más estable, sin grandes variaciones estacionales, con una aparente mayor presencia en otoño que decrece lentamente hasta el verano.

En primavera y verano hay un menor número de especies, pero con una mejor representatividad relativa de estas que en otoño e invierno (ver diversidades Tabla 3 ). Las estaciones mas similares en cuanto a la coincidencia de especies, son primavera y verano y las menos similares, son otoño y verano (ver similitudes Tabla 3). Las estaciones que más se parecen en cuanto al perfil de densidades de los géneros más importantes, son invierno, primavera, verano. Otoño por su parte, se

Tabla 1. Frecuencia relativa (\%) del total de los taxa aislados según estación y cultivo(PZ y AA) . Los taxa en fondo gris representan los considerados de interés clínico

\begin{tabular}{|c|c|c|c|c|c|c|c|c|c|c|}
\hline \multirow[t]{2}{*}{ Taxa Fúngicos } & \multicolumn{2}{|c|}{ Otoño } & \multicolumn{2}{|c|}{ Invierno } & \multicolumn{2}{|c|}{ Primavera } & \multicolumn{2}{|c|}{ Verano } & \multirow[b]{2}{*}{$\mathbf{n}$} & \multirow[b]{2}{*}{$\% \mathrm{FR}$} \\
\hline & $\mathbf{P Z}$ & AA & PZ & AA & $\mathbf{P Z}$ & AA & $\mathbf{P Z}$ & AA & & \\
\hline Acremonium breve (Sukap. \& Thirum) W.Gams & & & & & & & 2 & & 2 & 0,04 \\
\hline Acremonium sp. & 1 & 1 & & & 1 & 1 & & & 4 & 0,08 \\
\hline Acremonium strictum W.Gams & 0 & & & 2 & & & 2 & & 4,04 & 0,08 \\
\hline Alternaria alternata (Fr.) Keissler complex & 32 & 34 & 58 & 32 & 99 & 58 & 87 & 62 & 462 & 9,27 \\
\hline Alternaria sp. & 4 & 3 & 5 & 2 & 7 & 5 & 8 & 7 & 41 & 0,82 \\
\hline Alternaria tenuissima (Kunze ex Pers.) Wilts. & 12 & 12 & 64 & 9 & 24 & 14 & 22 & 16 & 173 & 3,47 \\
\hline Arthrobotrys cladodes Drechsler var. cladodes & & 1 & & & & & & & 1 & 0,02 \\
\hline Aspergillus niger & 3 & & 1 & & & & & & 4 & 0,08 \\
\hline Aspergillus sidowii & 1 & & & & & & & & 1 & 0,02 \\
\hline Aureobasidium pullulans (de Bary) Arn.var. pullulans & 2 & 12 & & & 1 & 1 & & & 16 & 0,32 \\
\hline Aureobasidium pullulans (de Bary)Arn.var.melanigenum H.Nij. & 37 & 12 & 16 & 4 & 8 & 3 & 4 & 3 & 87 & 1,75 \\
\hline Bipolaris cynodontis (Marignoni) Shoemaker. & & 3 & 8 & & 20 & 7 & 5 & 10 & 53 & 1,06 \\
\hline Bipolaris ellisii (Dinquah) Alcorn & 1 & & & & & & & & 1 & 0,02 \\
\hline Botrytis cinerea Pers. ex Nocca \& Balb. & & & & & 2 & & & & 2 & 0,04 \\
\hline Chaetomium bostrychodes Zopf & & & 1 & & & & & & 1 & 0,02 \\
\hline Chaetomium sp. & & & & & & & 1 & & 1 & 0,02 \\
\hline Chalara hughesii Nag Raj \& Kendrick & & & 4 & 1 & & & & & 5 & 0,10 \\
\hline Chalara sp. & & & & & & & & 2 & 2 & 0,04 \\
\hline Chaetopsina fulva Rambelli & 2 & 6 & 3 & 5 & & 3 & 1 & 4 & 24 & 0,48 \\
\hline Choanephora cucurbitarum (Berkeley \& Ravenel) Thaxter & & 1 & 13 & & & & & & 14 & 0,28 \\
\hline
\end{tabular}




\section{Continuación Tabla 1}

\begin{tabular}{|c|c|c|c|c|c|c|c|c|c|c|}
\hline Cladorrhinum anam. Cercospora samala Undagawa \& Muroi & & & & & & & 2 & & 2 & 0,04 \\
\hline Cladosporium cladosporioides (Fres.) de Vries & 116 & 132 & 136 & 72 & 133 & 67 & 98 & 81 & 835 & 16,75 \\
\hline Cladosporium oxysporum Berk. \& Curt. & 88 & 114 & 117 & 44 & 112 & 60 & 66 & 50 & 651 & 13,06 \\
\hline Cladosporium sp. & 2 & 3 & 4 & 1 & 3 & 2 & 4 & 2 & 21 & 0,42 \\
\hline Cladosporium sphaerospermum Penz. & 10 & 12 & 14 & 7 & 13 & 6 & 8 & 7 & 77 & 1,54 \\
\hline Clonostachys compactiuscula (Sacc.) Hawksworth \& W.Gams & 9 & 6 & 2 & & 1 & & & & 18 & 0,36 \\
\hline Clonostachys rhizophaga (Theon \& Jacobs) Schoers & & & & 2 & & & & & 2 & 0,04 \\
\hline Clonostachys rogersoniana Schroers (similis)? & 1 & & & & & & & & 1 & 0,02 \\
\hline Clonostachys rosea (Link: Fr.) Schroers et al. & 20 & 26 & 5 & 6 & 2 & & 1 & 3 & 63 & 1,26 \\
\hline Codinea britannica M.B.Ellis & 5 & & 14 & 13 & 2 & 9 & & 2 & 45 & 0,90 \\
\hline Codinea fertilis Hughes \& Kendrick & & & & & & & & 1 & 1 & 0,02 \\
\hline Curvularia clavata Jain & 4 & 23 & 1 & & & & 2 & & 30 & 0,60 \\
\hline Curvularia eragrostidis (Tsuda \& Ueyama)Sivanesan & & & & & & & 4 & 4 & 8 & 0,16 \\
\hline Curvularia lunata (Wakker) Boedijn & 1 & & 4 & 4 & 26 & 4 & 19 & 24 & 82 & 1,65 \\
\hline Curvularia lunata (Wakker) Boedijn var. aerea M.B.Ellis & & & & & 2 & 1 & 3 & & 6 & 0,12 \\
\hline Curvularia pallescens (Tsuda \& Ueyama)Sivanesan & & & 1 & & & & & & 1 & 0,02 \\
\hline Curvularia senegalensis (Speg.) Subarm. & & & 2 & & & & & & 2 & 0,04 \\
\hline Curvularia verruculosa Tandom \& Bilgrami ex M.B.Ellis & & & 3 & & & & & & 3 & 0,06 \\
\hline Cytospora chrysosperma Pers.:Fr. & & & & & & & 1 & & 1 & 0,02 \\
\hline Dactilaria sp. & & & & & 1 & & & & 1 & 0,02 \\
\hline Epicoccum nigrum Link & 39 & 23 & 63 & 36 & 103 & 52 & 60 & 65 & 441 & 8,85 \\
\hline Exserohilum rostratum (Drechsler) Leonard \& Suggs & & & 1 & & & & & & 1 & 0,02 \\
\hline Fusarium chlamydosporum Wollenw. \& Reinking & & 2 & & & & & & & 2 & 0,04 \\
\hline Fusarium oxysporum Schlecht.:Fr. & 9 & 2 & & & & & & & 11 & 0,22 \\
\hline Fusarium semitectum sensu Wollenw. & 19 & 23 & 16 & 17 & 45 & 7 & 66 & 22 & 215 & 4,31 \\
\hline Fusarium solani (Mart.) Sacc. & 3 & 4 & & 2 & & & & & 9 & 0,18 \\
\hline Fusarium sp. & 5 & & & & & & & & 5 & 0,10 \\
\hline Fusarium verticillioides (Sacc.) Niremb. & 1 & & & 2 & & & & & 3 & 0,06 \\
\hline Gelasinospora novoguineensis Tukada & & & & & 4 & & & & 4 & 0,08 \\
\hline Glomerella cingulata (Stonem.) Spauld.\& Schrenk & 3 & 2 & 1 & & & & & & 6 & 0,12 \\
\hline Harzia velata (Onions \& D.Jones) Hol.-Jech. & & & 1 & 5 & 42 & & 33 & 16 & 97 & 1,95 \\
\hline Harzia verrucosa (Togn.) Hol.-Jech. & & & 5 & 5 & & & & & 10 & 0,20 \\
\hline Hormonema dematioides Lagerberg \& Melin & & & 4 & & & & & 1 & 5 & 0,10 \\
\hline Leptosphaeria sp. 2 & & & & & & & & 2 & 2 & 0,04 \\
\hline Leptosphaeria sp.1 & & & 4 & & & & & & 4 & 0,08 \\
\hline Leptosphaerulina chartarum Roux. & & & & & & & 1 & & 1 & 0,02 \\
\hline Lophodermium pinastri (Schrad.:Hook) Chev. & 41 & 54 & 21 & 23 & 13 & 20 & 4 & 9 & 185 & 3,71 \\
\hline Metarrhizium anisopliae (Metschn.) Sorok. & & & & & 1 & & & & 1 & 0,02 \\
\hline Micelio sin fructificar & 22 & & 5 & 12 & 5 & 12 & & 19 & 75 & 1,50 \\
\hline Micosphaerella sp. & & & & & & & & 7 & 7 & 0,14 \\
\hline Microsphaeropsis olivacea (Bonord.) Höhn. & 1 & & 3 & 2 & & & & 2 & 8 & 0,16 \\
\hline Microsphaeropsis sp. & & & & & 1 & & & & 1 & 0,02 \\
\hline Monacrosporium sp. & & & & 2 & & & & & 2 & 0,04 \\
\hline Mortierella parvispora Linnemann & & & 2 & & & & & & 2 & 0,04 \\
\hline Mucor hiemalis Wehmer $f$. hiemalis & & 4 & 2 & 1 & 1 & & 5 & 1 & 14 & 0,28 \\
\hline Myrothecium graminum Lib. & & & 1 & 4 & 4 & & 4 & 6 & 19 & 0,38 \\
\hline Myrothecium indicum Rama Rao & & & & & & & 1 & & 1 & 0,02 \\
\hline Myrothecium jollymannii Preston & & & & & & & 1 & & 1 & 0,02 \\
\hline Myrothecium roridum Tode : Steudel & 1 & 2 & & & 14 & 1 & 12 & 2 & 32 & 0,64 \\
\hline Myrothecium verrucaria (Alb. \& Schw.) Ditm.:Fr. & & & & 2 & 2 & & & & 4 & 0,08 \\
\hline
\end{tabular}


Continuación Tabla 1

\begin{tabular}{|c|c|c|c|c|c|c|c|c|c|c|}
\hline Nigrospora anamorph of Khuskia oryzae Hudson & & & & & 5 & & & & 5 & 0,10 \\
\hline Nigrospora panici Zimm. & & & & & & & 2 & & 2 & 0,04 \\
\hline \begin{tabular}{|l} 
Nigrospora sacchari (Speg.) Mason \\
\end{tabular} & & & 16 & 6 & 3 & & & & 25 & 0,50 \\
\hline Nigrospora sphaerica (Sacc.) Mason & 37 & 8 & 11 & 2 & 28 & 24 & 23 & 24 & 157 & 3,15 \\
\hline Nodulisporium sp. & & 4 & & & & & & & 4 & 0,08 \\
\hline Ochroconis constricta (Abbott) de Hoog & & & & & & & & 2 & 2 & 0,04 \\
\hline Ochroconis tshawytschae (Doty \& Slater) Kirilenko \& Al-Achmed & & & 2 & 4 & 5 & 6 & & 6 & 23 & 0,46 \\
\hline Paecilomyces lilacinus (Thom) Samson & & & & & & & 1 & 4 & 5 & 0,10 \\
\hline Paecilomyces variotii Bain. & 1 & & & & & & & & 1 & 0,02 \\
\hline Penicillium citrinum Thom & 1 & & & & & & & & 1 & 0,02 \\
\hline Penicillium glabrum (Wehmer) Westling & 2 & & & & 1 & & & 1 & 4 & 0,08 \\
\hline Penicillium herquei Bain. \& Sart. & 2 & & & & & & & & 2 & 0,04 \\
\hline Penicillium purpurogenum Stoll & & & & & & & 5 & 4 & 9 & 0,18 \\
\hline Penicillium spp. & 8 & 2 & 5 & & & & & 3 & 18 & 0,36 \\
\hline Periconia byssoides Pers.:Mérat & & & 2 & & & & 1 & 1 & 4 & 0,08 \\
\hline Periconia cookei Mason \& M.B.Ellis & & & & & 1 & & & 2 & 3 & 0,06 \\
\hline \begin{tabular}{|l} 
Pestalotiopsis maculans (Corda) Nag Raj \\
\end{tabular} & 17 & 19 & 38 & 42 & 27 & 28 & 32 & 25 & 228 & 4,57 \\
\hline Pestalotiopsis sp. & & & 1 & & & & & & 1 & 0,02 \\
\hline Phoma eupirena Sacc. & & 5 & & & & & & & 5 & 0,10 \\
\hline Phoma glomerata (Corda) Wollenw. \& Hochapfel & & 3 & & & & & & & 3 & 0,06 \\
\hline Phoma herbarum Westend. & 1 & 6 & 4 & & 15 & 2 & 12 & 3 & 43 & 0,86 \\
\hline Phoma leveillei Boerema \& Bollen & & & 4 & & & & 2 & & 6 & 0,12 \\
\hline Phoma medicaginis Malbr. \& Roum var. medicaginis & 1 & & 15 & 4 & 4 & 5 & 2 & 3 & 34 & 0,68 \\
\hline Phoma sp. & 1 & 2 & 4 & 7 & 5 & 3 & 1 & 7 & 30 & 0,60 \\
\hline Pithomyces chartarum (Berk. \& Curt.) M.B.Ellis & 7 & 3 & 11 & 2 & 2 & 5 & 14 & 25 & 69 & 1,38 \\
\hline Podospora anserina (Ces.) Niessl & & & & & & & & 1 & 1 & 0,02 \\
\hline Polyscytatum fecundissimum Riess & & & 2 & & & & & & 2 & 0,04 \\
\hline Pullospora macrospora Nag Raj & & 1 & 9 & & & & & & 10 & 0,20 \\
\hline Ramichloridium schulzeri & & & & & & & & 1 & 1 & 0,02 \\
\hline Rhinocladiella spinifera (Nielsen \& Conant) de Hoog & & & & & 1 & & & & 1 & 0,02 \\
\hline Rhizopus oryzae Went \& Prinsen Geerligs & & & & & & & 5 & & 5 & 0,10 \\
\hline Rhizopus stolonifer (Ehrenb.:Fr.) Vuill. & & & 2 & & & & 4 & & 6 & 0,12 \\
\hline Sarcopodium macalpinei (Agnohothrudu \& Barua) Sutton & 1 & & & & & & & & 1 & 0,02 \\
\hline Sordaria fimicola (Rob.) Ces. \& de Notaris & & & 4 & & & & 3 & 1 & 8 & 0,16 \\
\hline Sphaeropsis sapinea (Fr.) Dyko \& Sutton & 3 & 2 & 3 & & 4 & 7 & 3 & & 22 & 0,44 \\
\hline Sporormiella minima Auersw., Ahmed \& Cain & & 4 & & & & & & & 4 & 0,08 \\
\hline Stauronema cruciferum (Ell.)H. P.Syd. \& Butler & & & & & & 1 & & & 1 & 0,02 \\
\hline Thielavia sp. & & 1 & & 1 & & & & & 2 & 0,04 \\
\hline Thozetella tocklaiensis (Agnohothrudu) Pirozynski \& Hodges & & & & & & & & 4 & 4 & 0,08 \\
\hline Torula herbarum Pers.:Gray & & & & & 1 & & & 3 & 4 & 0,08 \\
\hline Tryblidiopycnis pinastri Höhn. & & & & & 2 & 6 & & & 8 & 0,16 \\
\hline Trichoderma harzianum Rifai & 32 & 26 & 4 & 2 & 4 & & 12 & 11 & 91 & 1,83 \\
\hline Trichoderma koningii Oudemans & 24 & 16 & & & 6 & 9 & 14 & 4 & 73 & 1,46 \\
\hline Trichoderma longibrachiatum Rifai & & & 17 & 7 & 18 & 13 & 12 & 16 & 83 & 1,67 \\
\hline $\begin{array}{l}\text { Trichoderma spp. } \\
\text {. }\end{array}$ & 5 & 3 & 5 & 3 & & & 10 & & 26 & 0,52 \\
\hline Trichoderma viride Pers.:Fr. & 1 & & 8 & 6 & & & 1 & & 16 & 0,32 \\
\hline Trichotecium roseum (Per.) Link:Gray & & 1 & 3 & 4 & 5 & & 1 & & 14 & 0,28 \\
\hline \begin{tabular}{|l} 
Ulocladium atrum Preuss \\
\end{tabular} & & & & & & 1 & 1 & & 2 & 0,04 \\
\hline TOTALES & 639 & 623 & 775 & 407 & 829 & 443 & 688 & 581 & 4984 & \\
\hline Totales taxas potencialmente patógenos & 451 & 466 & 557 & 256 & 668 & 336 & 540 & 428 & 3702 & \\
\hline
\end{tabular}


Tabla 2.-Relaciones entre perfiles temporales de los 5 grupos de taxa más frecuentes ( A.- Alternaria, B.- Cladosporium, C.- E.nigrum, D.- Fusarium, E.- Trichoderma)

$$
\text { B C } \quad \text { D } \quad \text { E }
$$

A $107,4 \mathrm{p}<, 0012,5$ (NS) $55,2 \mathrm{p}<, 001 \quad 103,0 \mathrm{p}<, 001$

B $\quad 58,9 \mathrm{p}<, 00135,3 \mathrm{p}<, 001 \quad 24,9 \mathrm{p}<, 001$

C $\quad 32,9 \mathrm{p}<, 001 \quad 67,0 \mathrm{p}<, 001$

D

9,9 (NS)

Tabla 3.- Diversidad de Shannon Winner, similitud de Jaccard y relaciones entre estaciones

Num. Divers. (H) Simil. (Jacc.) Relación $\left(\mathrm{X}^{2}\right)$ spp. Absol. Relat Inv. Pri. Ver. Inv. Pri. Ver.

$\begin{array}{lrrrrrlrrr}\text { Otoñ. } & 30 & 2,23 & 7,4 \% & 0,5 & 0,5 & 0,44 & 65,6 & 166,9 & 96,2 \\ \text { Invi. } & 28 & 2,34 & 8,4 \% & & 0,53 & 0,5 & & 26,8 & 39,1 \\ \text { Prim. } & 14 & 2,32 & 16,6 \% & & & 0,64 & & & 38,6 \\ \text { Ver. } & 14 & 2,11 & 15,1 \% & & & & & \end{array}$

manifiesta con el perfil de densidades más alejado de las otras tres estaciones (ver relaciones Tabla 3).

Las otras especies de interés clinico que presentaron frecuencias de presencia superiores al $1 \%$ fueron, Bipolaris y Curvularia, con la mayor presencia en verano y primavera de B.cynodontis y C.lunata. Nigrospora sphaerica sólo disminuyó en invierno, mientras Phoma herbarum fue bastante estable en los meses de primavera y verano, diminuyendo hacia el invierno (Tabla 1).

\section{DISCUSION}

Los 2 medios de cultivo empleados en metodología, permitieron detectar la presencia de un alto número de taxa, en especial en agar papa zanahoria por el aporte de substancias simples de fácil asimilación para la mayoría de las especies fúngicas. Al ser un medio pobre que de por si reduce el tamaño de las colonias, la inclusión de Diclhoran, disminuyó más aún su tamaño y la cantidad de micelio, faciltando los recuentos sobre las acículas. Además permitió una buena y rápida esporulación en la mayoría de los taxa, atenuando en cierta medida el efecto inhibidor en algunos taxa de los compuestos fenólicos presente en las acículas. La mayor lentitud en el crecimiento de las colonias sobre el substrato en agar agua con Dichloran, permitió detectar algunos taxa substratos específicos, sin una gran disminución en la presencia de los principales géneros más frecuentes. Blak \& Dix (1976), comentan que Trichoderma viride, Mucor hiemalis y las especies de Penicillium, parecen estimularse frente a los compuestos fenólicos, sin embargo, esta situación sólo se observó e $T$. viride, debido a que Penicillium y los Mucorales fueron escasamente representados.

La zona muestreada, subtropical húmeda, no pre- senta grandes variaciones climáticas, permitiendo detectar con un solo muestreo estacional una considerable diversidad en la composición de especies, a pesar del corto período de incubación (3 semanas), con un listado de éstas seguramente incompleto y representado mayoritariamente por organismos de rápida esporulación y con altas capacidades saprofíticas competitivas interespecíficas. La composición de esta comunidad muestra un buen equilibrio, muchas especies esporádicas y un reducido número de especies dominantes en sus primeros estadios de la colonización como: Cladosporium, Alternaria, Epicoccum, Trichoderma y Fusarium. La mayoría de las especies que integraron estos géneros, se presentan también en la filósfera de otros vegetales y sus roles saprofíticos competitivos en las hojas caidas se ha detectado en varios tipos de literas ricas en fuentes de energía aprovechable como carbohidratos y hemicelulosa, (Kendrick \& Burges, 1962; Tubaki,1973; Blak \& Dix, 1976 -1977; Cabral, 1985; Takayuki \&Tokumasu,1995; Osono \& Takeda, 2002; Takashi Osono, 2002).

El método empleado y la corta incubación no permitió diferenciar, salvo excepciones (Lophodermium pinastri) el rol de epífito o endófitos de algunos taxa, muchas especies pueden colonizar ambas partes de este heterogéneo substrato en el tiempo, comportándose como saprófitos primarios o débiles parásitos, tal es el caso de las principales especies con las mayores tasas de presencia y constancia en el tiempo: Cladosporium cladosporioides, C.oxysporum, Alternaria alternata, Epicoccum nigrum, Pestalotiopsis maculans, Fusarium semitectum, Lophodermium pinastri, Nigrospora sphaerica, Trichoderma harzianum, Aureobasidium pullulans var. melanigenum, Curvularia lunata y Pithomyces chartarum, muchos de ellos comunes también en la litera herbácea (Bisset \& Parkinson 1980:Takashi, 2002). Son los colonizadores primarios, pero posteriormente reemplazados en el tiempo por un largo número de especies saprófitas secundarias que colonizan la litera después de la caída de las hojas, acorde a factores climáticos e interaciones específicas, sin descartar que varias especies dominantes pueden encontrarse en las sucesiones en el horizonte 0 de la litera de las coníferas en un único sitio geográfico ( Tokumasu \& Aoiki, 2002). Los factores determinantes en la composición de especies epífitas son generalmente la humedad y temperatura, ambos fluctúan según la estación y pluviosidad. Alternaria, Epicoccum, Fusarium y Rhizopus tienen correlación positiva frente a la temperatura y negativa con humedad, mientras Cladosporium parece no tener ninguna correlación con ambos parámetros y Phoma no se correlaciona con la temperatura. Estas situaciones coinciden con el trabajo de la composición taxonómica de hongos anemófilos relacionados con factores climáticos de Marchisio et al.(1997), a 
pesar de las diferencias geográficas. C.cladosporioides y C. oxysporum fueron las 2 especies con mayor presencia en todas las estaciones (dominantes), el primero, es la especie tipo de la nueva sección Hormodendropsis de Cladosporium (David,1997). Es un taxa extremadamente ubicuo, prevalente como saprófito en el suelo, litera y la superficie foliar de las plantas, productos manufacturados, así como uno de los más comunes hongos anemófilos en todas las latitudes (Domsch et al., 1980; Al-Doory, 1984; Aira et al., 2005).Su presencia constante junto a las otras 2 especies en todas las estaciones del año, sugiere que no guarda una estrecha relación con la mayor pluviosidad en primavera-verano ni con las menores temperaturas invernales. Además su capacidad de crecer sobre substratos de diversa naturaleza y ser un colonizador primario tolerante al estrés, le confieren gran capacidad saprofítica competitiva, cualidades que comparte en buena medida con las especies de Alternaria, en especial las integrantes del complex Alternaria alternata ( Pereira et al. 2002).

C.oxysporum, pertenece al subgénero Cladosporium y a la sección Cladosporium, considerada una especie final dentro de $\boldsymbol{C}$.herbarum aggr. (David ,1997), ampliamente disperso en regiones tropicales y subtropicales, generalmente como saprófito (más raramente como patógeno) en substratos herbaceos y leñosos diversos (Mckemy \& Morgan-Jones, 1991).

En nuestro trabajo pudimos observar cierta relación entre el clima y la composición de la micota solo en la distribución de especies más frecuentes o dominantes, sin embargo, se confirma que la distribución y la riqueza de especies observada guarda cierta relación con la mayor temperatura y humedad (alta pluviosidad) en los meses de verano y primavera de la zona muestreada. No se incluyeron en el análisis a otros taxa comunes en coníferas con un comportamiento similar en algunas estaciones como Lophodermium pinastri y Pestalotiopsis maculans que a pesar de que sus frecuencias, fueron en conjunto superiores al 8\%, no se dispersan por el viento y no tienen un interés clínico.

Los hongos filamentosos,en sus formas anamorfas cumplen roles saprotrofos mayoritariamente en ambientes de vida exógena, integrando las comunidades fúngicas más frecuentes en los suelos y la cobertura vegetal asociada. Muchos de ellos presentan grandes capacidades competitivas y antagónicas en la colonización y descomposición de una amplia variedad de substratos en todos los climas y latitudes. La condición de tolerantes al estrés cualidad común para los principales taxa aislados, les permiten sobrevivir y dispersarse con rapidez en substratos simples o complejos y cuando las condiciones ambientales son favorables algunos adquieren dominancia, pudien- do afectar no sólo a la agricultura (fitopatógenos), sino a la industria, los procesos biotecnológicos, las prácticas agrícolas y forestales y la salud humana y animal (Scholtof, 2003).

Nuestro análisis de la micota de interés clínico en la litera vegetal de coníferas, es un buen ejemplo de las capacidades de algunos grupos fúngicos en la competencia por un substrato difícil de descomponer, donde los integrantes de sólo 5 taxa comunes ejercieron dominancia estacional, representando cuantitivamente los 2/3 de toda la comunidad total. Sin embargo, algunos de estos colonizadores primarios, tienen un genoma que bajo ciertas circunstancias puede adaptarse al oportunismo o a la patogenia en animales de sangre caliente. Ambas cualidades necesitan un set de sutiles grados de patogenicidad, tipos de formas celulares, colonización y rutas de invasión fúngica, que en conjunto sean capaces de causar leves o severas micosis cuando el sistema inmune de los vertebrados está debilitado, pero raramente en un hospedador inmunocompetente. El potencial patogénico es complejo, muchas veces específico de un determinado organismo y es imposible aún llegar a conclusiones generales especialmente en sus atributos moleculares (Odds, et al., 2001; Van Burik \& Magee, 2001; Naglik et al., 2003)

En los 2 últimos decenios, muchos hongos dematiáceos e hialinos saprofíticos o con cierto grado de interés fitopatológico, han sido la causa de enfermedades en el hombre con presentaciones clínicas poco comunes (de Hoog et al., 2000).

Los hongos filamentosos dematiáceos, un heterogéneo grupo de organismos ampliamente dispersos en la naturaleza, poseen en común la producción de pigmentos de melanina y en la actualidad se han hecho frecuentes (más del 30\% de las micosis) como causa de enfermedad en el hombre (de Hoog et al.,1992, 2001; Fothergill, 1996; Norton et al., 1996; Groll \& Walsh, 2001). Las micosis causadas por estos hongos son llamadas comunmente faeohifomicosis (Ajello, 1975), pero se consideran también las cromoblastomycosis y los micetomas eumicóticos. Los taxa fúngicos de interés clínico fueron en su mayoría dematiáceos $(61,5 \%)$ representando además la mayor presencia del grupo en el susbtrato (84,6\%). Entre los más frecuentes oportunistas patógenos detectados en nuestra investigación se incluyen las especies de: Alternaria, Bipolaris, Cladosporium, Curvularia, Epicoccum, Nigrospora y Phoma, organismos ampliamente dispersos en el ambiente, suelo, madera y restos de material vegetal diverso en descomposición. Las infecciones cutáneas, subcutáneas, hiatrogénicas, transplantes de órganos, alérgicas y de córnea, entre otras, se presentan en todo el mundo, pero comunmente en los países tropicales y subtropicales, mediante implantación traumática o inhalación, pero no siempre en los individuos con algún 
grado de compromiso inmune. Estos hongos han sido la causa de sinusitis alérgica o invasivas pero, en la actualidad, las micosis diseminadas a varios órganos van en aumento en la literatura (Ebright et al., 1999;De Hoog et al., 2001;Lyke, et al., 2001; García-Díaz \& Baumgarten,2002; Brandt \& Warnock, 2003; Everett et al., 2003).

Debemos destacar en los individuos atópicos, que en el ambiente de bosques de coníferas, es posible la sensibilización in situ con propágulos fúngicos de la litera dispersos en el aire en ciertas épocas estacionales, especialmente en personal forestal, o habitantes de regiones cercanas. Es cada vez más frecuente encontrar en la literatura (niños y adultos), no solo casos de alergia por hongos dematiáceos de los géneros Alternaria, Bipolaris, Cladosporium, Curvularia, Epicoccum, Phoma y Nigrospora; sino cuadros clínicos más complejos asociados a la dispersión a distancia de sus mitosporas, capaces de la invasión de los senos nasales, paranasales, córnea, transplantes de órganos o aún infecciones del sitema nervioso central, en pacientes con o a veces sin compromiso inmmune (Muralidhar \& Sulthana, 1997; Chakrabarti \& Sharma, 2000; Arshad et al., 2001;Mezzari, et al., 2003; Tessari et al.,2003; Carter \& Boudreaux, 2004; Taj-Aldeen et al., 2004).

Varios autores han purificado y caracterizado los principales alergenos en Curvularia lunata y Epicoccum nigrum, tales como glicoproteínas, serino proteasas y proteínas de diferentes $\mathrm{kDa}$ y la gruesa reactividad con otros hongos dematiáceos y hialinos aeroalergenos, todos ellos frecuentes en nuestro trabajo (Bisht et al., 2002-2004; Grupta et al., 2004).

Los hongos filamentosos oportunistas hialinos, son también un grupo heterogéneo ampliamente disperso en la naturaleza que se consideran clínicamente como comunes alergógenos, toxicogénicos o productores de hialohifomicosis (de Hoog et al.,2000; Samson et al.,2004). En nuestro ambiente no fueron mayoritarios, destacándose sólo las especies de Fusarium y Trichoderma, mientras las especies de Aspergillus, Penicillium y Paecilomyces tuvieron una escasa presencia al igual que los Mucorales. Dentro del género Fusarium debe destacarse la alta presencia de $\boldsymbol{F}$. semitectum (= F. incarnatum) en todas las estaciones y en especial las más cálidas, sobrepasando ampliamente a todos los demás integrantes presentes del género. Esta situación puede asociarse a su amplia distribución ecológica en zonas cálidas y tropicales. Existen pocas referencias en la literatura sobre su rol patogénico en humanos, salvo en casos de queratitis, lesiones en piel quemada, endocarditis, cancer hematológico y su habilidad de producir micotoxinas citotóxicas (de Hoog et al., 2000; Nucci et al., 2003; Naiker \& Odhav, 2004). Su oportunismo es también escaso en animales y se ha detectado en hospedadores muy diversos, como bovinos (enfisema pulmonar agudo) y tortugas (invadiendo la queratina de su caparazón) (Linnabary \& Tarrier, 1988; Rose et al., 2001). Altomare et al.(2000), pudieron aislar 2 tipos de alfa pironas desde cultivos de arroz contaminado con $\boldsymbol{F}$. semitectum, que mostraron una considerable actividad antifúngica frente a hongos fitopatógenoso micotoxigénicos (Alternaria alternata, Ascochyta rabiei, Aspergillus flavus, Botrytis cinerea, Cladosporium cucumerinum, Phoma tracheiphila y Penicillium verrucosum), pero no con levaduras y bacterias encontradas en esta Poacea. Mostraron también buena actividad inhibitoria hacia los agentes de micosis humanas (Aspergillus y algunas Candida spp.). La producción de estos compuestos en las acículas por $\boldsymbol{F}$. semitectum puede considerarse como un factor de competitividad y antagonismo in situ en la litera, con seguridad de efecto limitante para algunos integrantes de la comunidad, que junto con la producción de mesoconidios secos, le permite una fácil dispersión anemófila y colonización de otros substatos, involucrándose en un rol alergógeno no despreciable. F. semitectum, tuvo baja constancia y abundancia en acículas de pino senescentes en una región oceánica subtropical en Japón (Tokumasu \& Aoiki, 2002)

Las especies de Fusarium son generalmente hongos del suelo de distribución mundial, grandes fitopatógenos y productores de diversas micotoxinas en cereales, de gran importancia en salud humana y animal por su acción cancerígena a largo plazo. F.oxysporum, F. solani y F. verticillioides, se reconocen como patógenos de mamíferos capaces de causar, queratitis, onicomicosis, infecciones en transplantes diversos y hialohifomicosis especialmente en personas con quemaduras (Guarro \& Gené, 1995; Hennequin et al., 1997; de Hoog et al., 2000). A pesar que la mayoría de las especies de Fusarium colonizan rápidamente diversos substratos con buena humedad, la presencia escasa de F.oxysporum, $\boldsymbol{F}$. solani y $\boldsymbol{F}$, chlamydosporum puede ser debida al tipo de substrato o la alta competencia.

Las especies de Trichoderma, de amplia distribución cosmopolita en suelos forestales y agrícolas, tuvieron una presencia casi constante en todas las estaciones, con 4 integrantes del género. Los integrantes de este taxa, fueron considerados en la literatura como microorganismos saprófitos con baja patogenicidad, pero en los últimos 2 decenios se ha reportado un aumento de varios tipos de infecciones causadas principalmente por T. Iongibrachiatum en pacientes con compromiso inmmune, siendo responsables de cuadros clínicos hematológicos malignos, en peritonitis asociadas a diálisis peritoneal, transplantes de órganos e infecciones pulmonares y cerebrales diseminadas(de Hoog et al., 2000; Groll \& Walsh, 2001; De Miguel et al.,2005). T. longibrachiatum, T.har- 
zianum, T. koningii, T. viride y T. pseudokoningi son las especies aisladas en diferentes cuadros clínicos (de Hoog et al.,2000). Las especies de Trichoderma, son conocidas por su uso potencial para el control biológico en hongos fitopatógenos ya sea por su micoparasitismo y producción de substancias antagónicas y celulasas de importancia biotecnológica (Samuels, 1996). Debido a la importancia clínica de este género y la capacidad de varias especies de crecer a $37^{\circ} \mathrm{C}$ en especial T.longibrachiatum, que crece bien a temperaturas de $40^{\circ} \mathrm{C}$, se han estudiado sus capacidades proteolíticas extracelulares en los aislamientos clínicos de esta cepa, determinándose que varias de sus isoenzimas, pueden estar asociadas en su rol de patógenos humanos facultativos (Kredics et al., 2004).

\section{CONCLUSIONES}

Se aislaron un total de 63 géneros fúngicos y 117 especies, mayoritariamente dematiáceos (81,9\%) y anamorfos de Ascomycota, con una mayor presencia en agar papa zanahoria que en agar agua.

Los taxa más frecuentes en ambos medios y esta- ción fueron en órden decreciente: Cladosporium spp. Alternaria spp., Epicoccum nigrum, Trichoderma spp. y Fusarium spp., representando el 65\% de la presencia total.

Las especie consideradas como oportunistas representaron más el $80 \%$ del total de la comunidad de las acículas de pino senescentes, predominando los taxas con varios tipos de conidiogénisis blástica (> 80\%), en especial las especies dematiáceas de Alternaria, Cladosporium, Epicoccum, Nigrospora, Curvularia y Bipolaris, las 3 primeras con dominancia y una buena constancia estacional en especial Alternaria spp. y Epicoccum nigrum. Las especies con conidiogénesis fialídica como Fusarium y Trichoderma, también tuvieron frecuencias relativas altas, destacando Fusarium semitectum y T.longibrachiatum, el primero por su alta presencia y el segundo por ser el más común en diversos cuadros clíni$\cos$

La constante dispersión anemófila de los conidios de estos taxa dominantes, reportados como alergénicos o con capacidades patogénicas, son de potencial interés médico para los habitantes de estas zonas boscosas que presenten algún grado de compromiso inmune.

\section{REFERENCIAS}

Ajello,L. (1975). Phaeohyphpomycosis: definition and etiology. Pan American Health Organization Sci. Publ. 304:126-133

Aira, M.J.; Jato, V.; Iglesias, I.; Rodriguez,F.J.; Méndez,J.; Albelda, Y.; Herves, M.; Piontelli, E.; Stchigel, A.M. (2005).Calidad del Aire, Polen y esporas en la comunidad Gallega. Xunta de Galicia.

Al-Doory, Y. (1984). Airborne fungi. In: Y. Al-Doory \& J.F. Domson (eds.) Mould allergy. Lea \& Fibiger, Philadelphia. pp.2740

Altomare,C.;Perrone,G.;Zonno,M.C.;Evidente,A.;Pengue, R.;Fanti,F.;Polonelli,L. (2000). Biological characterization of fusapyrone and deoxyfusapyrone, two bioactive secondary metabolites of Fusarium semitectum.J Nat Prod.63:1131-1135

Andrews, J.H. \& Hirano, S.S. (1991). Microbial ecology of leaves. Springer-Verlag, N.Y.

Arshad, S.H.; Tariq, S.M.; Matthews, S. \& Hakim, E. (2001). Sensitization to common allergens and its assocition with allergis disorders at age 4 years: a whole population birth cohort study. Pediatrics 80:33-39

Bills, G.F. \& Polishook, J.D. (1994). Abundance and diversity of microfungi in leaf litter of a lowland rain in forest in Costa Rica. Mycologia 86:187-198

Bisht, V.; Arora, N. Singh, B.P.; Gaur, S.N.; Sridhara,S. (2002). Antigenic and allergenic cross-reactivity of Epicoccum nigrum with other fungi. Ann. Allergy Asthma Inmunol. 89:285-291

Bisht, V.; Arora, N.; Singh, B.P.; Gaur, S.N.; Sridhara,S. (2004). Purification and characterization of a major cross-reactive allergen from Epicoccum purpurascens. Int. Arch. Allergy Inmunol. $133: 217-224$

Bisset,J. \& Parkinson,D.(1980). Long-term effects of fire on the composition and activity of the soil microflora of a subalpine, coniferous forest Can.J.Bot. 58:1704-1721

Black, R.L.B. \& Dix,N.J. (1976). Utilization of ferulic acid by microfungi from litter and soil. Trans. Br. mycol. Soc. 66:313-317

Black, R.L.B. \& Dix,N.J. (1977).Colonization of Scots pine litter by soil fungi. Trans. Br. mycol. Soc. 68:284-287

Brandt, M.E.\& Warnock, D.W. (2003). Epidemiology, clinical manifestations, and therapy of infections caused by dematiaceous fungi. J. Chemother. 15 (Suppl 2):S36-47

Cabral,D.(1985). Phyllosphere of Eucalyptus viminalis:Dynamics of fungal populations. Trans. Br.mycol. Soc.85:501-511

Carter, E. \& Boudreaux, C.(2004). Fatal cerebral phaeohyphomycosis due tu Curvularia lunata in a immunocompetent patient. J. Clin. Microbiol. 42:5419-5423

Chakrabarti, A. \& Sharma, S.C. (2000). Paranasal synus mycoses. Indian J. Chest. Dis. Allies Sci.42:293-304

David, J.C.(1997). A contribution to the systematics of Clados- 
porium. Revision of the fungi previously referred to Heterosporium. Mycological Papers 172.CAB International.

DeMiguel, D.; Gomez, P.; Gonzalez, R.; García-Suarez, J. Cuadros, J.A.; Banas; M.H.; Romanyk, J.; Burgaleta, C. (2005). Non fatal pulmonary Trichoderma viride infection in an adult patient with acute myeloid leukemia: Report of one case and review of literature. Diag. Microbiol. Infect. Dis. (PubMed) PMID :15994049

Domsch, K.H.; Gams, W. \& Anderson, T-H. (1980). Compendium of soil fungi,Vols. 1-2. Academic Press, London.

Ebright ,J.R.; Chandrasekar, P.H.; Marks, S.; Fairfax, M.R.; Aneziocoro, A.; McGinnis,M. (1999). Invasive sinusitis and cerebritis due to Curvularia clavata in a immunocompetent adult. Cl.Inf. Dis. 28:687-689

Ellis, M.B.(1971).Dematiaceous Hyphomycetes. CMI, Kew, Surrey. England

Ellis, M.B.(1976). More Dematiaceous Hyphomycetes. CMI, Kew, Surrey. England

Ellis, M.B. \& Ellis, J.P. (1985). Microfungi on Land Plants. An identification Handbook. Croom Helm, London \& Sydney

Everett, J.E.; Busick,N.P.; Sielaff, T.; Wahoff, D.C.; Dunn, D.L. (2003). A Deeply invasive Phoma species infections in a renal transplant recipient. Transplant. Proc. 35:1387-1389

Fothergill, A.W. (1996). Identification of dematiaceous fungi and their role in human disease. Cl. Inf. Dis. 22(Suppl 2):S179-184

Gams.W.\& Bisset, J. (1998). Morphology and identification of Trichoderma. In: C.P, Kubicek \& Harma, D.E.(Ed.). Trichoderma and Gliocladium. Basic biology, taxonomy and genetics, Vol.1. Bristol Pennsylvania, Taylor \& Francis Inc. pp.3-34

García-Díaz ,J.B. \& Baumgarten, K. (2002). Phaeohyphomycotic infections in solid organ transplant patient. Semin. Respir. Infect.17:303-309

Groll, H.A. \& Walsh, T.J. (2001). Uncommon opportunistic fungi: New nosocomial threats. Cl. Microbiol. Inf. Dis. 7 (Suppl. 2):S8-24

Groubière,F. (1974). Les champignon microcopiques liés aux aiguilles de sapin (Abies alba Mill.).2. Variation saisonnières de la microflore des aiguilles tombantes. Bulletin de la Societé mycologique de France. 90:325-333

Groubière,F. \&Debouzie,D. (2003). Local variation in microfungal population on Pinus sylvestris needles. Mycol. Res. 107:12211230

Grupta,R.; Sharma,V.; Sridhara,S.; Singh, B.P.; Arora, N. (2004).Identificationof serine protease as a major allergen of Curvularia lunata. Allergy 59:421-427

Guarro, J. \& Gené, J. (1995). Opportunistic Fusarial Infections in humans. Eur. J. Clin. Microbiol. Infect. 14:741-754

Hennequin,C.; Lavarde, V.; Poirot, J.L.; Rabodonirina,M.; Datry, A.; Aractingi, S.; Dupoy-Camet, J. et al.(1997). Invasive Fusarium infections:a retrospective survey of 37 cases. J. Med. \&Veter. Mycol. 35:107-114
Hoog,G.S.de.; Tan,G.S.; Stalpers, J.A. \& Stegehuis,G. (1992). The spectrum of opportunistic filamentous fungi present in the CBS culture collection. Mycoses 35:209-214

Hoog, G.S.de.; Guarro,J.; Gené, J. \& Figueras, M. (2000). Atlas of clinical fungi 2nd. edition. CBS,Utrecht,Netherland - Univ. Rovira i Virgili, Reus, Spain

Hudson, H.J. (1968). The ecology of fungi on plant remains above the soil. New Phytologist 67:837-874

Jones,E.B.G. \& Hyde, K.D. (2002). Succession: were do we go from here?. In: Fungal Succession (Eds. K.H.Hyde \& E.B.G.Jones) Fungal Diversity 10:241-253

Kendrick, W.B. \& Burges, A. (1962). Biological aspects of the decay of Pinus silvestris leaf litter. Nova Hedwigia 4:313-342

Klich,M.A.(2002). Identification of common Aspergillus species. CBS, Utrecht, The Nederland.

Kredics, L.; Antal, Z.; Scekeres, A.; Maczinger, L.; Doczi, I. Kevei, F.; Nagy, E. (2004). Production of extracellular proteases by human pathogenic Trichoderma longibrachiatum strains. Acta Microbiol. Immunol. Hung. 51:283-295

Like, K.E.; Miller, N.S.; Towne, L. \& Merz. W.G. (2001). A cutaneous ulcerative alternariosis: rare associationwith diabetes mellitus and unusual failure of itraconazol treatment. Clin. Infect. Dis. 32:1178-1187

Linnabary, R.D. \& Tarrier, M.P. (1988). Acute bovine pulmonary emphysema caused by the fungus Fusarium semitectum. Vet. Hum. Toxicol. 30:255-256

Mckemy,J.M. \& Morgan-Jones,G. (1991). Studies in the genus Cladosporium sensu lato.IV. Concerning Cladosporium oxysporum, a plurivorous, predominantly saprophytic species in warm climates. Mycotaxon 41:397-405

Marchisio,V.P.; Airaudi,D \& Barchi,C. (1997).One-year monitoring of the airborne fungal community in a suburb of Turin (Italy) and assessement of its functional relations with the environment. Mycol. Res. 101:821-828

Mezzari, A.; Perin, C.; Alves, S.; Guerra, L.A.B. Di Gesu, G.(2003). Os fungos anemófilos e sensibilizacao em indivíduos atópicos em Porto Alegre, RS. Rev. Assoc. Med. Bras. 49:270-273

Minter,D.W. (1981). Microfungi on needles, Twig and cones of pines in Czechoslovakia. Ceska Mycologie 35:90-101

Muller, C.S. (1974). Decomposition o f coniferous leaf litter. In: Biology of Plant Litter Decomposition Vol.1., C.H.Dickinson \& G.J.F.Pugh (eds.), Academic Press, London, UK. pp.105-128

Muralidhar,S. \& Sukthana, M. (1997). Nigrospora causing corneal ulcer: A case report. Indian J.Pathol. Microbiol. 40:549551

Nag-Raj,T.R.(1993). Coelomycetous anamorphs wuth appendagebearing conidia.Mycologue Publication,Waterloo, Ontario, Canadá.

Naglik,J.R.; Challacombe, S .J. \& Hube, B. (2003). Candida albicans secreted aspartil proteinase in virulence and pathogenesis. 
Microbiol and Mol. Biol.Reviews 67:400-428

Naiker, S. \& Odhav, B. (2004). Mycotic keratitis: profile of Fusarium species and their mycotoxins. Mycoses:47:50-56

Nelson, P.E.; Toussoun, T.A. \& Marasas, W.F.O. (1983). Fusarium species. An illustrated manual for identification. The Pennsylvania State University Press. University Park and London.

Norton R.S.; Cernoch P.L \& Daves J.R. (1996). Dematiaceous fungi are an increasing cause of uman disease. Clin Inf. Dis. 22:7380

Nucci, M.; Anaissie, E.J.; Queiroz-Telles, F.; Martins, C.A.; Trabasso, P., Solza, C.; Mangini, C. et al. (2003).Outcome predictors of 84 patients with hematologic malignancies and Fusarium infection. American Cancer Soc. 98:315-320

Odds,F.C.; Gow,N.AR.\& Brown, A.JP. (2001). Fungal virulence studies come of age. Genome Biology 2:review 1009.1-1009.4

Osono;T \& Takeda,H. (2002). Comparison of litter descomposing ability among diverse fungi in a cool temperate deciduos forest in Japan. Mycologia 94:421-427

Parkinson,D. (1982). Procedures for the isolation, cultivation and identification of fungi. In : Experimental Microbial Ecology, R.C.Burns \& J.H.Salter (ed.), Blackwell Scientific Publications. Oxford, U.K. pp.22-30

Pereira, P.T.; DE Carvallo, M,M.; Gírio,F.M.; Roseiro, J.M.; Amaral-Collaço, M.T. (2002). Diversity of microfungi in the phylloplane of plant growing in a Mediterranean ecosystem. J. Basic. Microbiol. 42:396-407

Pitt, J.I. (2000). A laboratory guide to common Penicillium species. Food Science Australia.

Rainer,A.D.M. (1994). Pattern-generating processesin fungal communities. In: Beyond the Biomass (K,Ritz; J.Dighton \& K.E. Giller, eds.). John Wiley \& Sons.Chichester. pp.247-258

Rose,F.L.; Koke, J.; Koehn, R. \& Smith, D. (2001). Identification of the etiological agent for necrotizing scute disease in the Texas tortoise. J.Wildlife Diseases 37:223-228

Samson, R.A.; Hoekstra, E.S \& Frisvald, J.C. (2004). Introduction to food and airborne fungi. Sevent ed. CBS Utrecht.
Samuels, G.J.(1996). Trichoderma: a review of biology and systematics of the genus. Mycol.Res. 100:923-935

Scholthof, K-B,G. (2003). One stop in the furrow: Linkage between agriculture, plant pathology, and public health. Annu. Rev. Public Health 24:153-174

Sivanesan, A. (1987). Graminicolous species of Bipolaris, Curvularia, Drechslera, Exserohilum and their Teleomorph. Mycol. Pap. 158. CAB International Mycological Institute

Sutton, B.C.(1980). The Coelomycetes. Fungi imperfecti with Pycnidia Acervuli and Stromata. CMI, Kew, Surrey, England.

Takashi O. (2002).Phyllosphere fungi on leaf litter of Fagus crenata: Ocurrence, colonización and sucession. Can.J.Bot: 80:460469

Takayuki,A.\& Tokumasu,S.(1995). Dominance and diversity of the fungal communities on fir needles. Mycol. Res. 99:1439-1449

Taj-Aldeen, S.J.; Hilal, A.A. \& Schell, W.A. (2004). Allergic fungal rhinosinusitis: A report of 8 cases, Am.J. Otorlaryngol. $25: 213-218$

Tessari,G.; Forni, A.; Ferretto, R.; Solbiati, M.; Faggian, G.; Mazzucco,A.; Barba, A. (2003). Lethal systemic dissemination from cutaneous infection due to Curvularia lunata in a heart transplant recipient. J. Eur.Acad. Dermatol. Venereol. 17:440-442

Tokumasu, S. (1998). Fungal succession n pine needles fallen at different seasons:The succession of surface colonizer. Mycoscience $39: 417-423$

Tokumasu, S. \& Aoiki,T. (2002). A new approach to studing microfungal succession on decaying pine needles in an oceanic subtropical region in Japan. In: Fungal Succession (Eds. K.H.Hyde \& E.B.G.Jones) Fungal Diversity 10:167-183

Tubaki,K.(1973).Sucessive fungal flora on sterilized leaves in the litter of forest. II IFO Res. Commun. 6:27-49

Van Burik, J-A.H \& Magee, P.T. (2001). Aspects of fungal pathogenesis in humans. Annu. Rev. Microbiol. 55:743-772

Zak,J.C. \& Rabatin, S.C (1997). Organization and description of fungal communities. In: The Mycota,IV. Environmental and Microbial Relationship (D.T.Wicklow \& B.Södeström, eds.). Springer- Verlag, Berlin. pp.33-46 